\title{
Fundamentação teórica dos sistemas de amostragem de solo de acordo com a variabilidade de características químicas
}

\author{
Theoretical background of soil sampling systems according \\ to variability of chemical characteristics
}

\author{
André Guarçoni ${ }^{1 \ddagger}$, Víctor Hugo Alvarez V.² e Fabrício Moreira Sobreira ${ }^{3}$
}

\footnotetext{
${ }^{1}$ Instituto Capixaba de Pesquisa. Assistência Técnica e Extensão Rural (INCAPER). Rod. BR 262 km 94 Aracê. 29375-5000 Venda Nova do Imigrante, ES, Brasil.

* Autor responsable (guarconi@incaper.es.gov.br)

${ }^{2}$ Departamento de Solos, Universidade Federal de Viçosa, (UFV). Avenida PH Rolfs s/n, Campus Universitário. 36570-000 Viçosa, MG, Brasil.

3 Instituto Federal Catarinense (IFC), Campus Araquari. Rodovia BR 280 km 27. Cx Posta 21. 89245-000 Araquari. SC, Brasil.
}

\section{RESUMO}

Devido à elevada variabilidade de determinadas características químicas, a amostragem de solo é uma das mais importantes fontes de erro em um programa de correção e adubação. O objetivo dessa revisão foi analisar criticamente os fundamentos teóricos que definem os tipos de amostragem de solo utilizados para diferentes sistemas de cultivo, avaliando o grau de influência das variações de características químicas conferidas por cada situação. Confrontando informações dos trabalhos consultados, foi possível inferir que a estratificação do terreno em unidades de amostragem diminui a ocorrência de dependência espacial causada por fatores do ambiente associados às variações de longa distância (macrodependência), mas não impede a ocorrência de dependência espacial a curtas distâncias em locais dentro do próprio estrato (mesodependência). A opção de se utilizar a análise da amostra composta por unidade de amostragem (fertilidade média) ou os mapas de fertilidade do solo, para recomendação de corretivos e fertilizantes, devese especialmente a questões econômicas. A coleta de amostras simples de pequeno volume e obtenção de médias locais, ou a formação de amostras compostas a partir destas "amostras simples", tende a incrementar a efetividade e a confiabilidade dos mapas de fertilidade.

Palavras chave: variabilidade aleatória, variabilidade cíclica, fertilidade média, mapas de fertilidade.

Cita recomendada:

Guarçoni, A., V. H. Alvarez V. e F. Moreira Sobreira. 2017. Fundamentação teórica dos sistemas de amostragem de solo de acordo com a variabilidade de características químicas. Terra Latinoamericana 35: 343-352.

\section{SUMMARY}

Due to high variability of chemical characteristics, soil sampling is one of the major sources of error in a correction and fertilization program. This critical review aimed to analyze the theoretical foundations that define the soil sampling types used for different cropping systems, assessing the degree of influence of variations in chemical characteristics conferred by each situation. Confronting information from the studies reviewed, we determined that the range of spatial dependence of soil chemical properties is dependent on the distance between previous sampling points. Land stratification in sampling units reduces the occurrence of spatial dependence caused by environmental factors associated with long-distance variations (macro-dependence), but does not prevent the occurrence of spatial dependence at short distances in positions within the stratum itself (meso-dependence). The option to use composite sample analysis from homogeneous field (average fertility) or the soil fertility maps for recommendation of lime and fertilizer, is primarily due to economic issues. The collection of single samples of small volume to calculate local averages, or the formation of composite samples from these "single samples" tends to increase the effectiveness and reliability of fertility maps.

Index words: random variability, cyclical variability, average fertility, fertility maps.

Recibido: septiembre de 2016. Aceptado: agosto de 2017. Publicado como revisión en Terra Latinoamericana 35: 343-352. 


\section{INTRODUÇÃO}

Para que o processo de amostragem de solo seja eficiente e econômico, deve-se conhecer e entender as causas da variabilidade das características de interesse do solo a ser amostrado, uma vez que essas irão influenciar o próprio programa de correção e adubação. Conforme a distribuição dessas características químicas no solo (aleatória ou sistemática), haverá uma forma mais indicada para o processo de amostragem (aleatória ou em grid) e para o embasamento da posterior recomendação de corretivos e fertilizantes (fertilidade média ou mapas de fertilidade).

Definindo-se que exista variabilidade aleatória de características químicas numa unidade de amostragem (UA) a ser cultivada (gleba ou talhão homogêneo), utiliza-se a amostragem aleatória de solo (probabilística), visando-se determinar a fertilidade média da mesma, para realização da correção do solo e adubação do cultivo, se necessário.

A individualização da UA é fundamentada, segundo Cantarutti et al. (2007), em indicadores de variação facilmente perceptíveis na área, como topografia, cobertura vegetal, tipo de solo, uso agrícola, condições de drenagem e produtividade das culturas. Por seu turno, a fertilidade média da UA é obtida analisando-se uma amostra composta, formada pela mistura homogênea de definido número de amostras simples. O número de amostras simples é calculado com base na estimativa da variabilidade de características do solo, determinada por meio de uma amostragem prévia da área (UA). $\mathrm{Na}$ prática, extrapola-se para áreas de cultivo comercial o número de amostras simples calculado para áreas experimentais.

Existe, contudo, o método de amostragem onde o local de coleta é tão importante quanto o número de amostras simples a ser coletado. Nesse caso, são formados grids de amostragem (amostragem sistemática) na área a ser cultivada, não havendo uma separação prévia da mesma em unidades de amostragem (UA). A partir da amostragem sistemática de solo (grids de amostragem), se for detectada, entre pontos do terreno, a ocorrência de dependência espacial para características químicas, mapas de fertilidade são elaborados para as áreas de cultivo, estimando-se valores intermediários entre os teores determinados nos locais de coleta (interpolação), por meio da análise geoestatística. De posse dos mapas de fertilidade, a aplicação de fertilizantes é realizada em taxa variável, visando homogênea disponibilização de nutrientes na área de cultivo.

A estimativa da variabilidade (Coeficiente de Variação - CV), considerando a variabilidade aleatória de características, depende de como é conduzida a amostragem para sua detecção, ou seja, o CV de determinada característica do solo pode aumentar ou diminuir de acordo com três fatores básicos, definidos por Upchurch e Edmonds (1991): local de coleta dentro da UA, volume e número de amostras simples a serem coletadas. Logo, esses fatores determinam a representatividade da amostra composta, no caso da variabilidade aleatória, e, em virtude da forma como são combinados, podem proporcionar uma amostragem de solo mais eficiente e econômica.

O objetivo dessa revisão foi realizar uma análise crítica dos fundamentos teóricos que definem os tipos de amostragem de solo utilizados para diferentes sistemas de cultivo, avaliando o grau de influência das variações de características químicas conferidas por cada situação.

\section{DESENVOLVIMENTO}

\section{Fertilidade Média do Solo e Mapas de Fertilidade}

Definir um valor médio para determinadas características da fertilidade do solo é um requerimento comum em estudos de campo (McBratney e Webster, 1983). Dentre esses, pode-se relacionar a recomendação de corretivos e fertilizantes.

Muitas fontes de erros potenciais estão envolvidas em um programa de adubação e, devido à elevada variabilidade de determinadas características químicas, uma das mais importantes é a amostragem de solo (Mallarino, 1996). O número ótimo de amostras simples para compor uma amostra composta representativa, adequada ao programa de adubação, irá depender da variabilidade da população amostrada, da importância em se estimar a média da população, do desvio desejado em torno dessa média e, principalmente, dos custos da amostragem e das análises laboratoriais (Mulla e McBratney, 2000).

Os solos são formados pela ação conjunta do clima, de organismos e do tempo, em determinado material de origem, numa definida posição da paisagem (Mulla e McBratney, 2000). Para esses autores, mesmo em 
uma área restrita, aparentemente homogênea, é pouco provável que existam dois locais que tenham recebido a mesma combinação ou interação de fatores de formação do solo. Isso justifica a classificação proposta por James e Wells (1990), relativa à variabilidade horizontal de características do solo, de acordo com a distância na qual ocorre: macrovariações $(>2 \mathrm{~m})$, mesovariações $(5 \mathrm{~cm}$ a $2 \mathrm{~m})$ e microvariações $(<5 \mathrm{~cm})$.

Além da intrínseca variabilidade na matriz do material de origem, pequenas mudanças na topografia podem afetar o fluxo e o armazenamento de água, que é o principal agente dos processos de formação do solo. $\mathrm{O}$ acúmulo de nutrientes e a deposição de material orgânico em alguns pontos, tendo como consequência a proliferação de organismos, podem gerar intensas variações a curta distância no solo. Esse fato foi relatado por Van Den Hende e Cottenie (1960), Jackson (1970), Alvarez e Carraro (1976), Saraiva et al. (1992), Raun et al. (1998), Guarçoni et al. (2006; 2007), que observaram elevadas diferenças para características químicas a curtas distâncias no solo.

É de grande relevância, no entanto, identificar a forma como a variabilidade de características do solo ocorre no campo, uma vez que irá influenciar o próprio processo de amostragem. A variabilidade dessas características pode correr de duas formas no campo: com dependência espacial ou aleatória. Quando uma característica do solo varia continuamente, numa direção específica, seguindo um gradiente, diz-se que tem dependência espacial ou correlação espacial e pode ser mapeada por meio da geoestatística (Burrough, 1991). Quando a variabilidade não pode ser ligada a uma causa conhecida e não varia continuamente, numa direção específica, ela é definida como aleatória (Upchurch e Edmonds, 1991).

A recomendação de corretivos e fertilizantes pela fertilidade média do solo é baseada na estatística paramétrica, ou seja, como premissa deve haver distribuição aleatória das características avaliadas e independência dos erros residuais dentro das UA, para que sejam calculados, sem introdução de uma tendência, além da média, o desvio padrão e o coeficiente de variação. Assim, para a formação de uma amostra composta representativa de uma gleba a ser cultivada (UA), esta não deveria, a princípio, apresentar dependência espacial para as características a serem avaliadas. De forma diversa, Brus e Gruijter (1997) relatam que as estratégias de amostragem aleatória são válidas também para áreas com dependência espacial, uma vez que a independência entre pontos seria criada pelo desenho de amostragem, ou seja, a forma de amostragem criaria independência entre pontos amostrados por meio da aleatorização.

Considerando a amostragem de solo sistemática, para Nanni et al. (2011), Molin et al. (2012) e Cherubin et al. (2014), a constatação de dependência espacial num campo de cultivo depende da escala de observação, mais precisamente, da distância entre os pontos de coleta das amostras simples que serão utilizadas na análise variográfica.

Essa constatação é apresentada na seguinte sequência de trabalhos de amostragem. Num experimento foram coletadas 206 amostras, na profundidade de $0-20 \mathrm{~cm}$, utilizando um grid regular de $50 \times 50 \mathrm{~m}$, em um Latossolo Vermelho eutroférrico, textura muito argilosa, cultivado por mais de 30 anos com cana-de-açúcar. Foram determinados valores de $\mathrm{pH}\left(\mathrm{H}_{2} \mathrm{O}\right)$ (Souza et al., 2004) e teores de P disponível (Marques et al., 2008). Os dados foram avaliados por meio da geoestatística, e foram observados alcances de 330 e $711 \mathrm{~m}$ para $\mathrm{pH}$ e $\mathrm{P}$ disponível, respectivamente. Segundo os autores, o valor do alcance de uma característica do solo garante que todos os pontos vizinhos, situados dentro de um círculo com esse raio, são tão similares que podem ser utilizados para estimar valores em qualquer ponto entre eles. Neste caso, consideraram a distância mínima de $700 \mathrm{~m}$ para que ocorra independência entre os teores avaliados nos locais amostrados dentro do campo de cultivo.

Salviano et al. (1998) coletaram amostras de solo na profundidade de $0-20 \mathrm{~cm}$, em uma malha de $5 \times 5 \mathrm{~m}$, numa área com Crotalaria juncea L. e observaram alcances de 22 e $12 \mathrm{~m}$ para $\mathrm{pH}\left(\mathrm{H}_{2} \mathrm{O}\right)$ e $\mathrm{P}$ disponível, respectivamente. Isso caracteriza uma distância de amostragem prévia dez vezes menor e alcances 15 e 59 vezes menores para $\mathrm{pH}$ e $\mathrm{P}$, respectivamente, em relação aos trabalhos de Marques et al. (2008) e Souza et al. (2004) citados anteriormente.

Paz González et al. (2000) coletaram amostras numa malha de $2 \times 2 \mathrm{~m}$ numa área cultivada e observaram alcances de $7 \mathrm{~m}$ para $\mathrm{pH}\left(\mathrm{H}_{2} \mathrm{O}\right)$ e $\mathrm{P}$ disponível. Em relação ao trabalho de Salviano et al. (1998), isso significa uma redução na amostragem prévia de 2.5 vezes e redução no alcance de 3 e 1.7 vezes, para pH e P disponível.

Yang et al. (2001) estimaram a variabilidade de $\mathrm{pH}\left(\mathrm{H}_{2} \mathrm{O}\right)$ em amostras indeformadas de solo medindo $10 \times 2.5 \mathrm{~cm}$ de lado e $10 \mathrm{~cm}$ de profundidade. Para 
tanto, os autores determinaram o $\mathrm{pH}$ a cada $1 \mathrm{~cm}$ das amostras, após saturação com água deionizada, utilizando um microelétrodo de $1 \mathrm{~mm}$ de diâmetro. Nesse caso, observaram dependência espacial dos valores de $\mathrm{pH}$, medida por meio de semivariograma, com alcances variando de $3.5-4.5 \mathrm{~cm}$. Esse resultado é muito marcante. Em relação ao trabalho de Salviano et al. (1998) já citado, há uma redução de 500 vezes na distância da amostragem prévia e uma redução no alcance de $\mathrm{pH}$ de 489 vezes, se for considerado o alcance de $4.5 \mathrm{~cm}$. Já em relação ao trabalho de Paz González et al. (2000), houve uma redução de 200 vezes na distância de amostragem e as mesmas 200 vezes no alcance de $\mathrm{pH}$, se for considerado o alcance de $3.5 \mathrm{~cm}$.

Apesar dos distintos solos, cultivos e regiões, a análise comparativa desses trabalhos indica que há uma clara diminuição no alcance da dependência espacial detectada, decorrente da redução na distância da amostragem, proposição esta já corroborada por trabalhos como os de Nanni et al. (2011), Molin et al. (2012) e Cherubin et al. (2014). Tal fato provém da capacidade de detecção da amostragem. A distância entre pontos de coleta irá promover a detecção de dependências especiais causadas por distintos fatores responsáveis pelas variações de características do solo. Isso afetará o alcance da dependência espacial, se esta existir.

Coletas de amostras de solo mais distantes entre si irão detectar dependências espaciais causadas por fatores de formação do solo que incidem de forma gradual sobre grandes áreas, como diferentes materiais de origem e clima. Por outro lado, a amostragem a curtas distâncias irá detectar a dependência espacial provocada por estratos geomorfológicos (posição na paisagem), classes de solo e o próprio manejo, ou seja, processos de adição, remoção e transformação que ocorram de forma mais localizada, formando gradientes ou variações cíclicas a curtas distâncias. Deve-se atentar para o fato de que os fatores apontados como causas das dependências espaciais a curtas distâncias são os mesmos considerados para separação das áreas em unidades de amostragem (UA), utilizadas na determinação da fertilidade média por meio da amostragem aleatória.

Fazendo um paralelo com a classificação de James e Wells (1990), na qual definem as macrovariações $(>2 \mathrm{~m})$ e as mesovariações (entre 0.05 e $2 \mathrm{~m}$ ) para a variabilidade aleatória, pode-se estabelecer as macro e as mesodependências espaciais, para o caso de haver dependência espacial entre características determinadas em distintos pontos de amostragem. A macrodependência espacial ocorreria entre pontos localizados em diferentes, mas contíguas, unidades de amostragem (UA), e seria caracterizada pela formação de gradientes contínuos de longa distância, permitindo a obtenção de maiores valores para o alcance de definida característica do solo. Assim, a macrodependência espacial seria detectada quando a distância de coleta de amostras for maior do que a área separada como uma Unidade de Amostragem (UA), a qual é baseada em indicadores de variação facilmente perceptíveis visualmente. Para sua detecção deve-se fazer uso de grids de amostragem de maior escala (upscale), como os propostos por Anderson et al. (2003).

A mesodependência ocorreria quando há dependência espacial entre pontos dentro de uma mesma UA, sendo caracterizada especialmente pela variação cíclica e com valores de alcance relativamente menores, podendo ser detectada por grids de amostragem de pequena escala (downscale-Anderson et al., 2003). Portanto, ao contrário da classificação proposta por James e Wells (1990) para a variabilidade aleatória dentro de uma UA, a classificação ora proposta para a dependência espacial não apresenta distâncias fixas quanto a separação de classes. Nesse caso, as distâncias são variáveis conforme o tamanho das UA demarcadas.

Em regiões onde as áreas de cultivo são homogêneas e as unidades de amostragem são extensas, a análise variográfica tende a estimar valores elevados de alcance para a dependência espacial, devido às macrodependências. Contudo, em áreas heterogêneas (regiões montanhosas, áreas de solos aluviais ou tabuleiros), onde as UAs apresentam dimensões reduzidas e a macrodependência é menor, serão estimados valores relativamente inferiores de alcance da dependência espacial.

A afirmação apoia-se em trabalhos como o de Montanari et al. (2008), que observaram maiores coeficientes de variação e menores alcances para a dependência espacial num latossolo de encosta convexa (UAs de menor tamanho), em relação a um argissolo, presente numa pedoforma mais plana (UAs de maior tamanho). Os autores atribuem o fato à maior continuidade espacial do Argissolo, gerada pela posição na paisagem. Souza et al. (2006) fizeram observações de mesmo teor, mas comparando áreas com pedoforma côncava (UAs de menor tamanho) e áreas com pedoforma plana (UAs de maior tamanho). 
O alcance devido às mesodependências (dentro das UAs) também varia, sendo proporcional ao tamanho das UAs, pois ficariam sempre restritos à área das mesmas. Trabalhos como os de Salviano et al. (1998), já mostravam que as características químicas do solo geralmente apresentam menor alcance entre pontos do que características físicas, como a granulometria. Se uma UA é individualizada, além de outros fatores, por mudanças de granulometria no solo, o trabalho dos autores citados corrobora a afirmação ora apresentada.

Essas observações levam à inferência de que dependências espaciais estejam ocorrendo tanto fora (macrodependências) quanto dentro (mesodependências) das unidades de amostragem, sendo sua detecção baseada na distância de amostragem utilizada na amostragem, upscale ou downscale, como sugerido por Anderson et al. (2003).

Para alguns estudiosos, essa constatação implicaria em abandono da clássica fórmula de Cline (1944) ${ }^{1}$,

$\mathrm{n}=\left(\mathrm{t}_{\alpha / 2} \mathrm{CV} / \mathrm{f}\right)^{2}$

apresentada sob tantas formas, por diversos autores, e utilizada para determinação do número de amostras simples necessárias à formação de uma amostra composta representativa da fertilidade média de uma UA. As recomendações de corretivos e fertilizantes pela fertilidade média do solo seriam consideradas pouco confiáveis, substituindo-as pelos mapas de fertilidade.

Quando não se detecta dependência espacial em determinada área, e pretende-se trabalhar com mapas de fertilidade, sugere-se uma amostragem mais detalhada para que se encontre a dependência entre os pontos (Molin et al., 2012). Assim, reduzindo o espaçamento nos grids de amostragem a dimensões inferiores as da UA, possibilita-se à detecção de mesodependências (dentro da UA) ao invés das macrodependências (fora da UA). Por outro lado, segundo Mallarino (1996), a redução no espaçamento dos grids pode gerar padrões cíclicos (tendências) de diferentes magnitudes, os quais, muitas vezes, afetam marcadamente a forma do semivariograma (modelo simples para o semivariograma pode não ser adequado), além de aumentar a ocorrência de "outliers" especialmente no sistema de plantio direto. Para Mulla e McBratney (2000), os "outliers" podem afetar significativamente a forma do semivariograma, diminuindo o alcance mesmo na presença de elevada dependência espacial.
A detecção das mesodependências (dentro da UA) tendem, portanto, a reduzir o alcance da dependência espacial e a confiabilidade dos mapas de fertilidade gerados, visando a recomendação de fertilizantes em taxas variáveis. No entanto, mesmo que exista dependência espacial dentro da UA, pode-se realizar a coleta de amostras simples para formação de uma amostra composta, visando a recomendação de corretivos e fertilizantes baseada na fertilidade média, uma vez que, para Cline (1944), a amostra composta de solo não é uma amostra da população (UA, gleba ou talhão homogêneo), pois sua única finalidade é estimar a fertilidade média, não podendo ser utilizada para qualquer outra estatística. Nesse sentido, Brus e Gruijter (1997) relatam que a amostragem aleatória é eficaz para determinar a média de uma característica do solo, desde que o número de amostras seja suficiente, pois o princípio de independência entre os pontos seria gerado pela própria aleatorização da coleta.

Por essas razões, pode-se indicar que nas UA o programa de recomendação de corretivos e fertilizantes é mais eficiente se for baseado na determinação da fertilidade média. Já para áreas maiores, que consideram várias unidades de amostragem (UA), a utilização dos mapas de fertilidade permite maior acurácia na recomendação, caso ocorra "macrodependência" espacial.

O trabalho de Anderson Cook et al. (1999) corrobora a afirmação anterior. Os autores utilizaram diferentes métodos de amostragem (três para elaboração de mapas de fertilidade-grid de $18.5 \times 30.4 \mathrm{~m}$, grid de 0.83 ha, grid de 0.33 ha e dois para determinação da fertilidade médiaamostra composta de toda a área e por tipo de solo (UA), visando recomendar adubações com $\mathrm{P}$ e $\mathrm{K}$, em duas áreas de cultivo. Observaram que a amostra composta por tipo de solo (UA) proporcionou a mais exata recomendação de fertilizantes quando a dependência espacial foi pequena. A diferença na quantidade total de fertilizantes recomendados, considerando os cinco métodos de amostragem, variou de 3 a 12\%. Relataram ainda que, a amostra composta por tipo de solo requer análise de poucas amostras e concorre com o grid de amostragem (mapa de fertilidade), mesmo quando ocorre elevada dependência espacial no campo. Os mesmos concluem que a amostragem por tipo de solo (UA) oferece significativas vantagens em termos de coleta de amostras e custos analíticos, melhorando a recomendação de fertilizantes em diversas situações.

${ }^{1} \mathrm{n}$ é o número de amostras simples para formar uma amostra composta representativa de área homogênea, $\mathrm{t}_{\mathrm{a} / 2}$ é o valor tabelado de distribuição $t$ de Student, $o$ qual depende do nível de probabilidade a e do número de graus de liberdade $(\mathrm{n}-1)$, CV é o coeficiente de variação da característica da fertilidade do solo a ser avaliada e f é o desvio admitido em torno da média. 


\section{Características da Amostra Simples de Solo}

Independente do sistema de amostragem, para Upchurch e Edmonds (1991) existem três fatores distintos envolvidos na amostragem de solo para uma característica particular: local de coleta, volume e número de amostras a ser coletado. O local de coleta irá depender da cultura, do tipo de solo e dos possíveis pontos de concentração de nutrientes no campo de cultivo. O local de coleta tem grande influência na estimativa da variabilidade da população (CV) (Zebarth et al., 1999; Alvarez e Guarçoni, 2003).

É de consenso na literatura que, ao se aumentar o tamanho da amostra (n), a estimativa da variabilidade vá diminuindo até atingir um valor mínimo, a partir do qual os decréscimos nessa estimativa são insignificantes em relação ao aumento do número de amostras (McBratney e Webster, 1983; Webster e Burgess, 1984; Burrough, 1991).

Guarçoni et al. (2007), trabalhando com amostras de diferentes volumes, para uma mesma profundidade de amostragem, observaram que essa lógica pode ser transposta para o volume das amostras simples. Assim, quanto maior o volume de solo coletado em cada amostra simples, menor é a estimativa da variabilidade e, consequentemente, menor o número de amostras simples necessário para formar uma amostra composta representativa de um talhão homogêneo, até um ponto mínimo onde a variabilidade se estabiliza e não adiantaria aumentar o volume das amostras simples. Esses autores realizaram amostragens de solo em três situações de preparo-coleta (plantio direto, plantio convencional antes da aração e plantio convencional após a aração) e, além de elaborarem modelos que definiam o número de amostras simples a ser coletado, em função do diâmetro do trado para cada situação de preparo-coleta, observaram que a estimativa da variabilidade da maioria das características químicas do solo avaliadas foi, de maneira geral, semelhante entre o plantio direto e o plantio convencional antes da aração, e menor no plantio convencional depois da aração, devido à manutenção de linhas de plantio nos dois primeiros casos.

O número de amostras simples necessárias à formação de uma amostra composta representativa irá depender da estimativa da variabilidade da população amostrada (CV) e da precisão requerida para se estimar a média da população (f), segundo a fórmula de Cline (1944): $\mathrm{n}=\left(\mathrm{t}_{\alpha / 2} \mathrm{CV} / \mathrm{f}\right)^{2}$

Nessa fórmula, o CV é obtido em uma amostragem prévia da área (UA). Portanto, o número de amostras simples a ser coletado é consequência clara dos locais de coleta e do volume das amostras simples utilizadas na amostragem prévia, uma vez que esses fatores influenciam diretamente a magnitude da variabilidade detectada (Guarçoni et al., 2007).

É importante ressaltar que, para cada forma e volume das amostras simples utilizadas na amostragem prévia, existirá um número ótimo de amostras simples a ser coletado, o qual irá conferir a mesma representatividade à amostra composta formada. Muitas vezes, a coleta de um maior número de amostras simples de pequeno volume gera um menor esforço de amostragem do que a coleta de um reduzido número de amostras simples de volume elevado (Guarçoni et al., 2007).

\section{Forma da Amostra Simples de Solo}

Considerando a profundidade de amostragem padrão de 0 a 20 ou 0 a $10 \mathrm{~cm}$, a área de abrangência da amostra simples de solo (dimensões horizontais) é aquela que proporcionará as diferenças no volume amostrado. Poucos são os trabalhos encontrados na literatura que comparam amostras simples com seções diferentes (retangulares, quadradas, circulares, etc.), uma vez que, para qualquer volume, será calculado um número de amostras simples que irá gerar uma amostra composta representativa, considerando o sistema de amostragem aleatória. Para Burrough (1991), o número de amostras simples é mais importante que sua localização ou seu volume. O autor está correto, mas faz essa afirmação sem considerar o tempo e o esforço necessário para se realizar a amostragem. O inverso, nesse caso, parece mais lógico, ou seja, para um número pré-definido de amostras simples, existe um volume de solo, considerando a mesma profundidade de amostragem, que gera amostras compostas mais representativas.

Para Schilindwein e Anghinoni (2002), o número de amostras deve contemplar as macrovariações (>2 m), enquanto a forma, o volume (área da amostra) e o local de amostragem devem contemplar as meso $(5 \mathrm{~cm}$ a $2 \mathrm{~m})$ e as microvariações $(<5 \mathrm{~cm})$. Esses autores compararam teores de $\mathrm{P}$ e $\mathrm{K}$ disponíveis, determinados ao se coletar amostras simples de diferentes volumes (áreas diferentes para uma mesma profundidade) e 
observaram que os coeficientes de variação (CV) dos teores de $\mathrm{P}$ e $\mathrm{K}$ diminuíram com o aumento da área das amostras simples, independentemente da forma da seção (retangular ou quadrada). Porém, não observaram diminuição substancial nos CV's com o aumento da área das amostras simples a partir de $5 / 10 \mathrm{~cm}$ (espessura/largura), demonstrando que, ao se aumentar a seção da amostra simples, a estimativa da variabilidade diminui até atingir um valor mínimo, a partir do qual, os decréscimos nessa estimativa são insignificantes em relação ao aumento da seção.

Essa tendência foi comprovada de forma mais detalhada por Guarçoni et al. (2007), que selecionaram modelos hiperbólicos para explicar a variabilidade de características químicas do solo em função do volume das amostras simples (diferentes áreas para uma mesma profundidade), constatando redução na variabilidade em função do aumento gradativo da área (mesma profundidade) das amostras simples de solo. Os autores relatam que a forma de resposta se deve à incorporação no volume de solo coletado das variações horizontais a curtas distâncias (microvariações), o que diminui, consequentemente, a estimativa da variabilidade.

Aárea de abrangência da amostra simples apresenta grande influência sobre o número de amostras necessário para formação de uma amostra composta, mas não a sua forma, podendo esta apresentar seção quadrada, retangular ou circular.

\section{Efeito da Variabilidade Cíclica sobre os Sistemas de Amostragem de Solo}

A concentração localizada de nutrientes é causada especialmente pela aplicação de fertilizantes em linha (James e Hurst, 1995), o que gera a variabilidade cíclica, advinda da detecção de pontos de alta e baixa concentração e um grande aumento na variabilidade horizontal de características químicas do solo (Mallarino, 1996), sendo esta uma característica do sistema de plantio direto (Anghinoni e Salet, 1998; Schlindwein e Anghinoni, 2000 e 2002).

A variabilidade cíclica é uma das dependências espaciais que podem ocorrer dentro de uma unidade de amostragem (UA), ou seja, seria uma das causas das mesodependências estabelecidas no presente artigo. No entanto, essa mesma mesodependência não ocorre apenas no sistema de plantio direto, pois pode estar presente de forma marcante também em UAs estabelecidas no plantio convencional. Segundo
Guarçoni et al. (2007), a manutenção de pontos de concentração pode ocorrer no plantio convencional antes de novo preparo do solo (aração/gradagem), sendo o padrão cíclico semelhante ao gerado no sistema plantio direto. Após a aração/gradagem, segundo esses autores, ocorre drástica redução na variabilidade cíclica antes observada. Os autores concluem que ações antrópicas nem sempre aumentam a variabilidade das características químicas do solo. Isso se dá, apenas, quando a ação se configura em aporte e, ou, concentração de nutrientes em determinados pontos do terreno.

Para reduzir o efeito da variabilidade cíclica causada pela aplicação em linha dos fertilizantes, visando diminuir o número de amostras simples para determinação da fertilidade média do solo, é de consenso na literatura que se deva utilizar um equipamento de coleta que incorpore estas variações na amostra coletada: a pá de corte (Anghinoni e Salet, 1998; Schlindwein e Anghinoni, 2000 e 2002; Oliveira et al., 2002; Alvarez e Guarçoni, 2003; Rosolem et al., 2010). Porém, a amostragem com pá de corte demora de 1.3 a 2.3 vezes mais do que com a utilização de trados (Rosolem et al., 2010), o que pode ocasionar um problema operacional.

Considerando o volume e o local de coleta das amostras simples de solo, como fatores responsáveis por aumentar a representatividade da amostra composta, Alvarez e Guarçoni (2003) compararam dois equipamentos de coleta (trado de caneca e pá de corte) quanto à detecção da variabilidade de características químicas do solo, em área sob plantio direto de milho. Os autores observaram que os teores de $\mathrm{K}$ disponível, $\mathrm{Mg}^{2+}$ trocável e os valores de $\mathrm{H}+\mathrm{Al}$ foram os únicos que diferiram ao se proceder a amostragem com pá de corte ou com trado de caneca. $\mathrm{Na}$ amostragem aleatória com trado de caneca a variabilidade de todas as características avaliadas e o número de amostras simples necessário à formação de uma amostra composta foram maiores do que na amostragem com pá de corte. Todavia, a amostragem localizada com trado ( $17 \%$ das amostras no sulco de plantio, $33 \%$ a $10 \mathrm{~cm}$ do sulco e $50 \%$ no ponto médio entre sulcos) e com pá de corte proporcionaram variabilidades semelhantes.

A variabilidade cíclica pode ainda dificultar a detecção de dependência espacial ou reduzir o alcance dessa dependência, quando for definida a utilização de amostragem sistemática (grids) para a elaboração de mapas da fertilidade do solo. Para esse caso, Cherubin 
et al. (2014) recomendam malhas de amostragem menores. Essas malhas menores têm a vantagem de manter a sensibilidade em relação às diferentes escalas de variabilidade espacial das características do solo, o que, para Bottega et al. (2013), é fundamental.

A redução nas malhas de amostragem muitas vezes é antieconômica (Raun et al., 1998) ou produz outliers (Mallarino, 1996) que afetam significativamente a forma do semivariograma, diminuindo o alcance mesmo na presença de elevada dependência espacial (Mulla e Mcbratney, 2000). Nesse caso, a variação cíclica pode ser atenuada com o uso do bulking (retirada de muitas amostras simples de pequeno volume e obtenção de médias locais, ou formação de amostras compostas a partir destas "amostras simples") (Webster e Burgess, 1984; Burrough, 1991; Cahn et al., 1994; Mulla e Mcbratney, 2000).

Considerando a proposta de bulking como ponto de partida, Guarçoni et al. (2006) definiram as dimensões do "Indivíduo Solo", conceituado como a menor área (considerando definida profundidade) na qual se deve amostrar o solo para caracterizar a fertilidade efetivamente explorada por uma planta ou por um grupo de plantas (fertilidade local média), sendo suas dimensões laterais grandes o bastante para incluir variações representativas a curtas distâncias na composição do solo (meso emicrovariações). Segundo os autores, a amostragem do "indivíduo solo", ao invés da coleta de amostras simples pontuais, pode atenuar a ocorrência de padrões cíclicos e de "outliers", melhorando a forma do semivariograma e aumentando o alcance. Além disso, a confiabilidade dos mapas de fertilidade pode ser aumentada, por estar sendo considerada a fertilidade local média. Estes benefícios podem ser conseguidos sem aumentar o número de amostras a serem analisadas, bastando para isso analisar a amostra composta representativa do "indivíduo solo".

Se o "indivíduo solo" é a menor área (considerando definida profundidade) na qual se deve amostrar o solo para caracterizar a fertilidade efetivamente explorada por uma planta ou por um grupo de plantas (fertilidade local média), a maior área a ser amostrada com o mesmo objetivo (fertilidade média) seria composta pela população de indivíduos, que nada mais é do que a própria unidade de amostragem (UA). Exatamente por isso, amostras simples coletadas fora da UA podem comprometer a representatividade da amostra composta formada, quando se tem por intuito determinar a fertilidade média do solo.

\section{CONSIDERAÇÕES F INAIS}

Os métodos de amostragem detectam a variabilidade de características químicas do solo de formas distintas. Some-se a isso, o fato de que os teores determinados nos locais amostrados no terreno podem apresentar dependência espacial ou não. Assim, determinados princípios e modelos amostrais podem gerar maior efetividade para a avaliação da fertilidade do solo, visando a recomendação de corretivos e fertilizantes.

Existem basicamente dois modelos de amostragem de solo, que necessitam do atendimento de algumas premissas: a amostragem aleatória e a amostragem sistemática. A amostragem aleatória se baseia na distribuição aleatória das variáveis e independência entre pontos do terreno, sendo fundamentada na estatística "clássica". Já na amostragem sistemática, há necessidade de detecção de dependência espacial entre características determinadas em pontos específicos do terreno, para que sejam elaborados, por meio da geoestatística, os mapas de fertilidade do solo.

Há um pensamento difundido de que a amostragem aleatória não é adequada para determinação da fertilidade média, pois, em geral, as características do solo são espacialmente dependentes, apresentando autocorrelação entre os pontos amostrados. Contudo, deve ficar claro que a fertilidade média da UA é determinada por meio da análise de uma amostra composta de solo, não havendo necessidade de qualquer outra estatística. Além disso, a própria amostragem aleatória pode gerar independência entre os pontos, se ela for realizada de forma realmente ao acaso, o que justificaria a determinação da fertilidade média do solo utilizando-se esse método de amostragem.

Considerando a ocorrência de dependência espacial no terreno, é apresentado o conceito de meso e de macrodependência espacial. As mesodependências seriam dependências espaciais que ocorrem dentro das Unidades de Amostragem (UA), e tem seu alcance limitado às dimensões das mesmas. As macrodependências espaciais seriam causadas por fatores de formação do solo que incidem de forma gradual sobre grandes áreas, como diferentes materiais de origem e clima, sendo detectadas por grids de amostragem mais espaçados (upscale).Nesse caso, a distância entre os pontos de coleta deve ser maior do que a área separada como uma Unidade de Amostragem (UA), a não ser que suas dimensões sejam grandes o suficiente para que as mesodependências sejam 
confundidas, na prática, com as macrodependências.

Para que a amostragem de solo seja eficaz, tornandose a base adequada para um bom programa de correção e adubação, pode-se trabalhar com dois sistemas: "unidades de amostragem/fertilidade média" ou "grids de amostragem/mapas de fertilidade". Se a opção for por trabalhar com unidade de amostragem/fertilidade média, o mais eficiente, inclusive economicamente, é determinar a fertilidade média das UAs por meio da amostragem aleatória, existindo mesodependência espacial ou não. Se a opção for pelos grids de amostragem/mapas de fertilidade, a amostragem sistemática e utilização da geoestatística é a opção mais eficiente, especialmente visando a detecção das macrodependências espaciais e a obtenção de maiores alcances.

Para qualquer sistema de amostragem, no entanto, o volume da amostra simples ou pontual irá influenciar na representatividade da amostra composta formada ou do entorno do ponto amostrado. Amostras com maior volume, especialmente com áreas maiores tendem a ser mais representativas, pois incorporam variações a curtas distâncias no material coletado (microvariações). Especialmente para a elaboração de mapas de fertilidade do solo, a amostragem em grid pode ser mais eficaz se forem utilizadas, em substituição às amostras pontuais, amostras compostas formadas por amostras simples coletadas em reduzidas subáreas (Indivíduo Solo), locadas nos pontos de interseção da malha de amostragem.

\section{REFÊRENCIA}

Alvarez V., V. H. e I. M. Carraro. 1976. Variabilidade do solo numa unidade de amostragem em solos de Cascavel e de Ponta Grossa, Paraná. Rev. Ceres 23: 503-510.

Alvarez V., V. H. e A. Guarçoni. 2003. Variabilidade horizontal da fertilidade do solo de uma unidade de amostragem em sistema plantio direto. Rev. Bras. Ciênc. Solo 27: 297-310. doi:10.1590/S0100-06832003000200010.

Anderson Cook, C. M., M. M. Allen, R. Noble, e R. Khosla. 1999. Phosphorus and potassium fertilizer recommendation variability for two mid-Atlantic Coastal plain fields. Soil Sci. Soc. Am. J. 63: 1740-1747. doi:10.2136/sssaj1999.6361740x.

Anderson, M. C., W. P. Kustas, and J. M. Norman. 2003. Upscaling and downscaling: A regional view of the soil-plant-atmosphere continuum. Agron. J. 95: 1408-1423.

Anghinoni, I. e R. L. Salet. 1998. Amostragem do solo e as recomendações de adubação e calagem no sistema plantio direto. pp. 27-52. In: N. J. Nuernberg (ed.). Conceitos e fundamentos do sistema plantio direto. Sociedade Brasileira de Ciência do Solo/ Núcleo Regional Sul. Lages, Brasil.
Bottega, E. L., D. M. Queiroz, F. A. Carvalho Pinto, e C. M. Alves de Souza. 2013. Variabilidade espacial de atributos do solo em sistema de semeadura direta com rotação de culturas no cerrado brasileiro. Rev. Ciênc. Agron. 44: 1-9.

Brus, D. J. e J. J. Gruijter. 1997. Random sampling or geostatistical modelling? Choosing between design-based and model-based sampling strategies for soil (with Discussion). Geoderma 80: $1-44$.

Burrough, P. A. 1991. Sampling designs for quantifying map unit composition. pp. 89-126. In: M. J. Musbach and L. P. Wilding (eds.). Spatial variabilities of soil and land forms. SSSA Spec. Publ. 28, SSSA. Madison, WI, USA.

Cantarutti, R. B., N. F. Barros, H. E. P. Martinez, e R. F. Novais. 2007. Avaliação da fertilidade do solo e recomendação de fertilizantes. pp. 769-850. In: R. F. Novais, V. H. Alvarez V., N. F. Barros, R. L. F. Fontes, R. B. Cantarutti, e J. C. L. Neves (eds). Fertilidade do solo. Sociedade Brasileira de Ciência do Solo. Viçosa, Brasil.

Cherubin, M. R., A. L. Santi, M. T. Eitelwein, D. R. Menegol, C. O. da Ros, O. H. de Castro Pias, e J. Berghetti. 2014. Eficiência de malhas amostrais utilizadas na caracterização da variabilidade espacial de fósforo e potássio. Ciênc. Rural 44: 425-43. doi:10.1590/S0103-84782014000300007.

Cline, M. G. 1944. Principles of soil sampling. Soil Sci. 58: 275-288. Guarçoni, A., V. H. Alvarez, R. Ferreira Novais, R. B. Cantarutti, H. Garcia Leite, e F. Morel Freire. 2006. Definição da dimensão do indivíduo solo e determinação do número de amostras simples necessário à sua representação. Rev. Bras. Ciênc. Solo 30: 943-954. doi:10.1590/S0100-06832006000600004.

Guarçoni, A., V. H. Alvarez V., R. Ferreira Novais, R. B. Cantarutti, H. Garcia Leite, e F. Morel Freire. 2007. Diâmetro de trado necessário à coleta de amostras num cambissolo sob plantio direto ou sob plantio convencional antes ou depois da aração. Rev. Bras. Ciênc. Solo 31: 947-959. doi: 10.1590/S010006832007000500012.

Jackson, M. L. 1970. Análisis química de suelos. Omega. Barcelona, España.

James, D. W. e K. L. Wells. 1990. Soil sample collection and handling: technique based on source and degree of field variability. pp. 25-44. In: R. L. Westerman (ed.). Soil testing and plant analysis. SSSA Book Series No. 3. Madison, WI, USA.

James, D. W. e R. L. Hurst. 1995. Soil sampling technique for bandfertilized,no-till fields with Monte Carlo Simulations. Soil Sci. Soc. Am. J. 59: 1768-1772.

Mallarino, A. P. 1996. Spatial variability patterns of phosphorus and potassium in no-tilled for two sampling scales. Soil Sci. Soc. Am. J. 60: 1473-1481. doi:10.2136/ sssaj1996.03615995006000050027x.

Marques Júnior, J., Z. M. Souza, G. T. Pereira, e D. Mazza Barbieri. 2008. Variabilidade espacial de matéria orgânica, P, K e CTC de um latos solo cultivado com cana-de-açúcar por longo período. Rev. Biol. Ciênc. Terra 8: 143-152.

McBratney, A. B. and R. Webster. 1983. How many observations are needed for regional estimation of soil properties? Soil Sci. 135: 177-183.

Molin, J. P., A. F. Colaço, E. F. Carlos, e D. Mattos Junior. 2012. Yield mapping, soil fertility and three gaps in an orange orchard. Rev. Brasil. Fruticul. 34: 1256-1265. doi: 10.1590/ S0100-29452012000400035. 
Montanari, R., G. T. Pereira, J. Marques Júnior, Z. M. Souza, R. J. Pazeto, e L. A. Camargo. 2008. Variabilidade espacial de atributos químicos em Latossolo e Argissolos. Ciênc. Rural 38: 1266-1272.

Mulla, D. J. and A. B. McBratney. 2000. Soil spatial variability. pp. A321-A352. In: M. E. Sumner, M. E. (ed.). Handbook of soil science. CRC Press. Boca Raton, FL, USA.

Nanni, M. R., F. P. Povh, J. A. M. Demattê, R. B. Oliveira, M. L. Chicati, and E. Cezar. 2011. Optimum size in grid soil sampling for variable rate application in site-specific management. Sci. Agric. 68: 386-392.

Oliveira, F. H. T., R. F. Novais, V. H. Alvarez V., R. B. Cantarutti, e N. F. Barros. 2002. Fertilidade do solo no sistema plantio direto. pp. 393-486 In: C. E. G. R. Schaefer, J. W. V. Mello, L. M. Costa, N. F. Barros, e V. H. Alvarez V. (eds.). Tópicos em ciência do solo. Sociedade Brasileira de Ciência do Solo. Viçosa, Brasil.

Paz-González, A., S. R. Vieira, and M. T. Taboada Castro. 2000. The effect of cultivation on the spatial variability of selected properties of an umbric horizon. Geoderma 97: 273-292. doi: 10.1016/S0016-7061(00)00066-5.

Raun, W. R., J. B. Solie, G. V. Johnson, M. L. Stone, R. W. Whitney, H. L. Lees, H. Sembiring, e S. B. Phillips. 1998. Microvariability in soil test, plant nutrient, and yield parameters in bermudagrass. Soil Sci. Soc. Am. J. 62: 683690doi: 10.2136/sssaj1998.03615995006200030020x.

Rosolem, C. A. T. S. Tozi, e R. A. Garcia. 2010. Amostragem de terra para fins de fertilidade em função da ferramenta de amostragem. Rev. Ceres 57: 405-414. doi: 10.1590/S0034737X2010000300018.

Salviano, A. A. C., S. R. Vieira, e G. Sparovek. 1998. Variabilidade espacial de atributos de solo e de Crotalariajuncea L. em área severamente erodida. Rev. Brasil. Ciênc. Solo 22: 115-122.

Saraiva, O. F., V. H. Alvarez, e L. M. da Costa. 1992. Variabilidade de algumas características físicas e químicas de um Podzólico Vermelho-Amarelo Câmbico distrófico. Rev. Ceres 39: 529-541. doi: 10.1590/S0100-06832007000300001.
Schlindwein, J. A. e I. Anghinoni, I. 2002. Tamanho da subamostra e representatividade da fertilidade do solo no sistema plantio direto. Ciênc. Rural 32: 963-9968. doi: 10.1590/S010384782002000600008 .

Schlindwein, J. A. e I. Anghinoni. 2000. Variabilidade horizontal de atributos de fertilidade e amostragem do solo no sistema plantio direto. Rev. Brasil. Ciênc. Solo 24: 85-91.

Souza, Z. M., J. Marques Junior, G. T. Pereira, e L. F. Moreira. 2004. Variabilidade espacial do $\mathrm{pH}, \mathrm{Ca}, \mathrm{Mg}$ e $\mathrm{V} \%$ do solo em diferentes formas do relevo sob cultivo de cana-deaçúcar. Ciênc. Rural 34: 1763-1771. doi: 10.1590/S010384782004000600015.

Souza, Z. M., J. Marques Júnior, G. T. Pereira, e R. Montanari. 2006. Otimização amostral de atributos de latossolos considerando aspectos solo-relevo. Ciênc. Rural 36: 829-836.

Upchurch, D. R. and W. J. Edmonds. 1991. Statistical procedures for specific objectives. pp. 49-71. In: M. J. Musbach and L. P. Wilding (eds.). Spatial variabilities of soil and landforms. SSSA Special Publication No. 28. Madison, WI, USA.

Van Den Hende, A. e A. Cottenie. 1960. L'estimation de la fertilité du sol par les méthodes chimiques nouvelles. Comp. Rend. Des Recherches. Travaux du Centre de Chemie Physique Agricole. Irsia. Bruxelles, Belgique.

Webster, R. and T. M. Burgess. 1984. Sampling and bulking strategies for estimating soil properties in small regions. J. Soil Sci. 35: 127-140. doi: 10.1111/j.1365-2389.1984.tb00267.x.

Yang, J. J., D. E. Mosby, S. W. Casteel, and R. W. Blanchar. 2001. Microscale $\mathrm{pH}$ variability for assessing efficacy of phosphoric acid treatment in lead-contaminated soil. Soil Sci. 166: 74-381.

Zebarth, B. J., M. F. Younie, J. W. Paul, J. W. Hall, and G. A. Telford. 1999. Fertilizer banding influence on spatial and temporal distribution of soil inorganic nitrogen in a corn field. Soil Sci. Soc. Am. J. 63: 1924-1933. doi: 10.2136/sssaj1999.6361924x. 